\title{
Influence of Turbulence in Aorta-like Tube: Computational and Experimental Study
}

\author{
J. Jagos ${ }^{1 \dagger}$, J. Kohut ${ }^{1}$, M. Kotek ${ }^{2}$, P. Skacel ${ }^{1}$ and J. Bursa ${ }^{1}$ \\ ${ }^{1}$ Faculty of Mechanical Engineering, Brno University of Technology, Brno, 616 69, Czech Republic \\ ${ }^{2}$ Department of Physical Measurement, Technical University of Liberec, Liberec, 461 17, Czech Republic
}

†Corresponding Author Email: 145427@vutbr.cz

(Received October 9, 2021; accepted February 3, 2021)

\begin{abstract}
The presented paper aims at comparison of modelling approaches to a pulsatile fluid flow in aorta-like tube; it investigates their influence on the shape of the velocity profiles and waveforms, and consequently on wall shear stress. Comparisons of computational results between rigid and compliant tubes with laminar and low Re turbulent models of fluid are presented. The results were validated with PIV experimental data through the velocity profile in the half-length section of the tube for both cases (rigid and compliant) and the overall agreement was very good, almost perfect for the rigid case. Frequency of the pulse pump in the experimental circuit was $1 \mathrm{~Hz}$, the diameter of the tube $\sim 20 \mathrm{~mm}$, and maximum deformation of the compliant tube during a period was $12 \%$. The turbulent model improved the agreement with the experimental data by flattening the velocity profiles in both cases, but the effect was much more pronounced for the compliant tube, especially during the deceleration phase. This work confirms the hypothesis stated by Brindise and Vlachos (2018) that a longer deceleration phase triggers transition to turbulence. We put foundations for extension of this hypothesis to compliant tubes where this conclusion was confirmed for physiological Reynolds and Womersley numbers. The main outputs of this study are: (i) the length of deceleration phase should be considered (in addition to the geometry or severity of stenosis) in decision whether fluid simulations should be performed with or without laminar flow assumption; (ii) for fluid simulations of blood vessels considering their compliance, a special care should be devoted to time synchronization between BCs to prevent unphysiological waveforms.
\end{abstract}

Keywords: Pulsatile flow; Pulse wave velocity; Compliant tube; Low Re turbulent model; Boundary condition.

\section{NOMENCLATURE}

$\begin{array}{ll}\text { BC } & \text { Boundary condition } \\ \text { BL } & \text { Boundary layer } \\ \text { CFD } & \text { Computational Fluid Dynamics } \\ \text { FSI } & \text { Fluid-Structure Interaction } \\ \text { PDMS } & \text { Polydimethylsiloxane } \\ \text { PIV } & \text { Particle image velocimetry }\end{array}$

\section{INTRODUCTION}

Not only the recent progress in computational performance has been quite outstanding, but also the level of numerical modelling in biomechanics has risen in a similar manner. For example, cf. 2D idealized stationary CFD simulations vs. 3D patientspecific transient simulations of fluid structure interaction (FSI). Regarding to the latter, the most time-consuming part of the whole workflow is no longer the computing itself, but segmentation, meshing and pre-processing protocols (Morris et al.

$\begin{array}{ll}\text { Re } & \text { Reynolds number } \\ \text { TKE } & \text { Turbulent kinetic energy } \\ \text { ITKE } & \text { Integrated turbulent kinetic energy } \\ \text { WSS } & \text { Wall shear stress } \\ & \\ \alpha & \text { Womersley number }\end{array}$

2017). Finding the optimal level of the features and simplifications of such models is crucial. It is believed that the blood flow in larger vessels like aorta or carotid bifurcation has a transitional character and a tendency to turbulences is further gaining significance in case of severe stenoses (Miyazaki et al. 2017; DiCarlo et al. 2019). Transitional flow occurs at subcritical Re numbers and needs thus an appropriate choice of a turbulence model. Blood flow has also a pulsating character, with frequency of $\sim 1 \mathrm{~Hz}$ in rest. Therefore, regarding to turbulence, no conclusion on the character of such flow can be drawn on the basis of 
Re number alone. The pulsatile character can be defined by another dimensionless parameter, Womersley number $\alpha$ (Mcdonald 1954). Many attempts have been made trying to connect these two dimensionless parameters into one and to find a critical value, above which the flow is not purely laminar, as summarized in (Xu and Avila 2018). Moreover, it was pointed out that turbulence occurs in a specific part of the period, namely in the deceleration phase, where the inverse pressure gradient plays a significant role in separation of the boundary layer. In a straight rigid tube, turbulent formation takes place in the near wall region and then it diffuses to the core region (Özahi and Çarpınlıoğlu 2017; Brindise and Vlachos 2018). This last reference emphasized also the importance of acceleration rate and duration of the deceleration phase in the transition to turbulence. A long deceleration phase induced an earlier onset of transition, while a shorter and more rapid deceleration delayed the transition. On the other hand, the acceleration phase, lower pulsation frequency and higher amplitude are attributed to stabilization effect, resulting in a laminar flow (Xu et al. 2017; Xu and Avila 2018). Nevertheless, despite the amount of studies dealing with the transition to turbulence in a pulsating flow, it still remains poorly understood and no solution merging all aspects has been provided (Xu and Avila 2018). Moreover, none of these works deal with the effect of wall compliance.

The presented study investigates the impact of turbulence on velocity profiles in rigid and compliant tubes. The aim of this study is therefore twofold: (i) to elucidate the effect of compliance on the onset of transition compared to the rigid tube case; (ii) on the basis of experimental results from PIV, to choose the suitable fluid modelling technique for such a complex flow.

Table 1 Dimensions of the tubes (see Fig. 1B).

\begin{tabular}{|c|c|c|}
\hline $\begin{array}{c}\text { Dimensions } \\
{[\mathrm{mm}] .}\end{array}$ & \multicolumn{2}{|c|}{ Cases } \\
\hline & Rigid & Compliant \\
\hline L1 & 30 & 300 \\
\hline L2 & 25 & 25 \\
\hline L3 & 450 & 450 \\
\hline r1 & 12.5 & 12.5 \\
\hline r2 & - & 11.25 \\
\hline r3 & 10 & 8.95 \\
\hline r4 & 7.5 & 7.5 \\
\hline
\end{tabular}

\section{Experimental Circuit}

The experimental circuit is presented in Fig. 1A, dimensions of the test region for the rigid and compliant tubes (Fig. 1B) are summarized in Table 1. The vertical arrangement of the test section prevents the compliant tube from bending and thus the results were almost symmetrical along its axial axis. Downward flow was chosen arbitrarily.

The working fluid of the circuit was water (at $20^{\circ} \mathrm{C}$ )

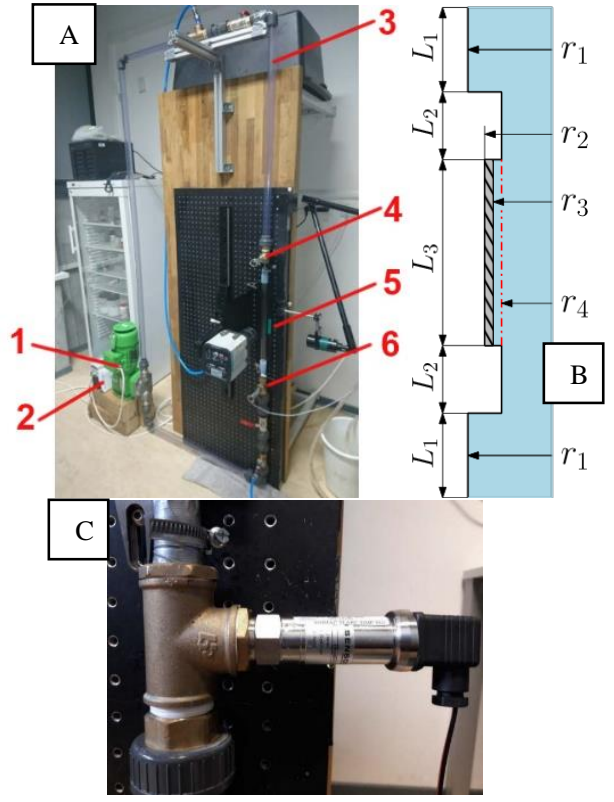

Fig. 1. (A) Experimental circuit: 1 - Membrane pump; 2 - Unidriver M200; 3 - Hydraulic circuit; 4 - Upper (input) pressure sensor 1; 5 - Test region; 6 - Lower (output) pressure sensor 2; (B) Schema of the test section;

(C) Detail of the pressure sensor.

with polyamide fluorescent seeding particles (coloured with Rhodamine B) having their emitting wavelength of $570 \mathrm{~nm}$, size of $5 \mu \mathrm{m}$ (small enough to follow the flow and not to disturb its Newtonian character), and the mass density comparable with water. The flow was actuated by the membrane pump Bran+Luebbe ProCam Smart DS500, controlled with the frequency driver Emerson Control Techniques Unidriver M200 to set the optimal pulsation rate of 1Hz. An upper water tank was connected to the circuit to set up the initial pressure in the circuit. Thereafter the tank was disconnected to avoid its impact on dumping of the pulsations. The flow was illuminated by a pulse Nd:YLF laser Litron LDY 302 (527nm wavelength). The PIV data was recorded using a system from Dantec Dynamics and its SpeedSense camera with sampling frequency of $1000 \mathrm{~Hz}$ synchronised with the laser. Such a high frequency gave us a detailed description of the pulsating flow and enabled us to capture eventual turbulent structures.

The PIV measurements were made in the measuring section illuminated by the laser sheet in the halflength of the tube. A dewarping method was used to process the dataset of images. Changes of refractive indices on interfaces between different matters (water, glass/PDMS, air) cause image distortion, which results in erroneous evaluation of the velocity fields, especially at the wall. Before the measurement, an image calibration was performed using a chessboard calibration target placed inside the tube and filled with water. Cross-Correlation method was used for analysis of the measurement datasets (using Interrogation Area of 32 px x 32 px, corresponding to $0.43 \mathrm{~mm} \times 0.43 \mathrm{~mm}$ ) and Moving Average Validation method for their evaluation 
(Averaging $3 \times 3$ cells of interrogation areas, without substituting the vectors and without validation on boundaries). Finally, velocity profiles in the halflength section of the tube were extracted from the velocity vector fields.

Pressure sensors with sampling frequency of $500 \mathrm{~Hz}$ were located at the inlet and outlet of the test region (the measured tube). The maximum overpressure of the cycle was set to $\sim 70 \mathrm{kPa}$ for the compliant tube (Fig. 2B). This value was several times higher than the systolic blood pressure, which enabled us to reach deformation comparable with human aorta because the PDMS material of the tube was stiffer than the aortic wall.
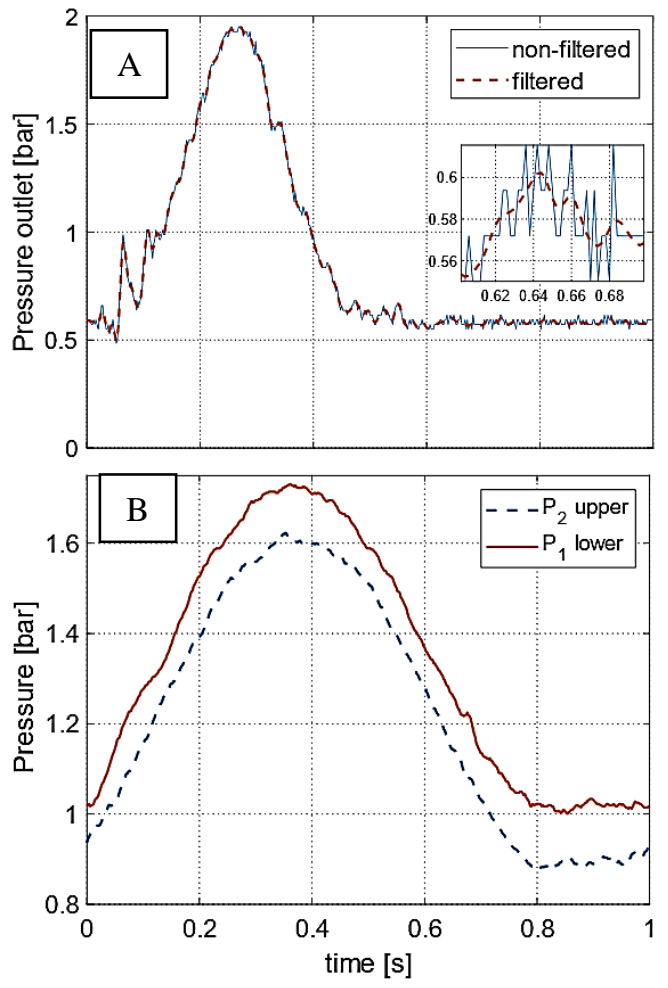

Fig. 2. (A) Transient absolute pressure profile from the lower sensor of the rigid tube. High frequency noise was filtered out by a low pass filter. (B) Transient absolute pressure profile from the upper and lower pressure sensors of the compliant tube after filtering out the high frequency noise.

\section{NUMERICAL SimUlations}

Numerical simulations were realized for both rigid and compliant tubes. In both cases, relative roughness of the tube (with respect to its diameter) was on the order of $10^{-5}$ or lower, thus the tubes were considered hydraulically smooth. The CFD simulations of the flow in the rigid tube were performed in ANSYS Fluent solver, and the FSI simulations of the compliant tube by coupling Fluent for the liquid phase with ANSYS Structural solver (based on Finite Element Method) for the solid phase. Due to axial symmetry, only a quarter of the test region (parts 4,5,6 in Fig. 1 incl. necessary lengths $\mathrm{L}_{1}$ of the hydraulic circuit ante and behind the test region) was considered in the computational model. Acceleration of gravity was taken into consideration because the tube in the circuit was in vertical direction.

Regarding the CFD simulations in detail, finite volume method was used with two governing equations. The law of conservation of momentum which is represented by Navier-Stokes Eq. (1) and continuity Eq. (2) (law of mass conservation). Equations stated below are for incompressible fluid with constant density $\rho$, both written in terms of Einstein summation convention. If the flow is purely laminar, the problem can be solved based on these Eqs.:

$$
\begin{aligned}
& \frac{\partial v_{i}}{\partial t}+v_{j} \frac{\partial v_{i}}{\partial x_{j}}=-\frac{1}{\rho} \frac{\partial p}{\partial x_{i}}+v \frac{\partial^{2} v_{i}}{\partial x_{j} \partial x_{j}}+g_{i} \\
& \frac{\partial v_{i}}{\partial x_{i}}=\frac{\partial v_{j}}{\partial x_{j}}=0
\end{aligned}
$$

Where $v_{i}$ and $v_{j}$ represent velocity $\left[\mathrm{m} \cdot \mathrm{s}^{-1}\right], t$ is time [s], $x_{i}$ and $x_{j}$ are coordinates [m], $p$ pressure [Pa], $\rho$ density $\left[\mathrm{kg} \cdot \mathrm{m}^{-3}\right], v$ kinematic viscosity $\left[\mathrm{m}^{2} \cdot \mathrm{s}^{-1}\right], g_{i}$ gravitational acceleration $\left[\mathrm{m} \cdot \mathrm{s}^{-2}\right], \frac{\partial v_{i}}{\partial t}$ local acceleration $\left[\mathrm{m} \cdot \mathrm{s}^{-2}\right], v_{j} \frac{\partial v_{i}}{\partial x_{j}}$ convective acceleration $\left[\mathrm{m} \cdot \mathrm{s}^{-2}\right], \frac{1}{\rho} \frac{\partial p}{\partial x_{i}}$ pressure term $\left[\mathrm{m} \cdot \mathrm{s}^{-2}\right], v \frac{\partial^{2} v_{i}}{\partial x_{j} \partial x_{j}}$ diffusion term $\left[\mathrm{m} \cdot \mathrm{s}^{-2}\right]$. However, for turbulent or transitional flow, the time-averaging is necessary and so-called Reynolds averaged Navier-Stokes Eq. (3) (RANS) are obtained. The instantaneous value of velocity is decomposed into time-averaged component $\bar{v}$ and fluctuating component $v^{\prime}$ (see Eq. (5)). This leads to an additional term $\overline{\rho v_{i}^{\prime} v_{j}^{\prime}}[\mathrm{Pa}]$ called Reynolds stress.

$$
\begin{aligned}
& \frac{\partial \bar{v}_{i}}{\partial t}+\partial \bar{v}_{j} \frac{\partial \bar{v}_{i}}{\partial x_{j}}=-\frac{1}{\rho} \frac{\partial \bar{p}}{\partial x_{i}}+v \frac{\partial^{2} \bar{v}_{i}}{\partial x_{j} \partial x_{j}} \\
& -\frac{\partial}{\partial x_{j}}\left(\overline{v_{i}^{\prime} v_{j}^{\prime}}\right)+g_{i}
\end{aligned}
$$

$\frac{\partial \bar{v}_{i}}{\partial x_{i}}=\frac{\partial \bar{v}_{j}}{\partial x_{j}}=0$

$v=\bar{v}+v^{\prime}$

The solution of RANS equations together with continuity Eq. (4) was closed with two equations model $k-\omega$ SST (see Eqs. (7) and (8)), so the Boussinesq hypothesis, Eq. (6), was included also. Meaning, turbulent viscosity $\mu_{t}[\mathrm{~Pa} \cdot \mathrm{s}]$ is assumed to be scalar with the turbulence being isotropic. Also, the optional correction for low $R e$ was enabled in program Fluent, since it is most likely that the flow is in transitional region. The correction is done by damping coefficient, which damps the turbulent viscosity $\mu t$. 


$$
\begin{aligned}
& \rho \overline{v_{i}^{\prime} v_{j}^{\prime}}=\mu_{t}\left(\frac{\partial \bar{v}_{i}}{\partial x_{j}}+\frac{\partial \bar{v}_{j}}{\partial x_{i}}\right) \\
& \frac{\partial}{\partial t}(\rho k)+\frac{\partial}{\partial x_{i}}\left(\rho k \bar{v}_{i}\right)=\frac{\partial}{\partial x_{j}}\left(\Gamma_{k} \frac{\partial k}{\partial x_{j}}\right) \\
& +G_{k}+S_{k}-Y_{k} \\
& \frac{\partial}{\partial t}(\rho \omega)+\frac{\partial}{\partial x_{i}}\left(\rho \omega \bar{v}_{i}\right)=\frac{\partial}{\partial x_{j}}\left(\Gamma_{\omega} \frac{\partial \omega}{\partial x_{j}}\right) \\
& +G_{\omega}+S_{\omega}-Y_{\omega}+D_{\omega}
\end{aligned}
$$

Where $k$ is turbulent kinetic energy $\left[\mathrm{m}^{2} \cdot \mathrm{s}^{-2}\right], \omega$ specific dissipation rate $\left[\mathrm{s}^{-1}\right], \quad \Gamma_{k}$ effective diffusivity of $k$ [Pa's], $\Gamma_{\omega}$ effective diffusivity of $\omega$ [Pass], $\widetilde{G_{k}}$ generation of $k$ by mean velocity gradients $\left[\mathrm{Pa} \cdot \mathrm{s}^{-1}\right], G_{\omega}$ generation of $\omega\left[\mathrm{Pa} \cdot \mathrm{m}^{-2}\right], Y_{k}$ dissipation of $k$ cause by turbulence [Pa's $\mathrm{s}^{-1}$ ], $Y_{\omega}$ dissipation of $\omega$ cause by turbulence $\left[\mathrm{Pa} \cdot \mathrm{m}^{-2}\right], S_{k}$ user defined term $\left[\mathrm{Pa} \cdot \mathrm{s}^{-1}\right], S_{\omega}$ user defined term $\left[\mathrm{Pa} \cdot \mathrm{m}^{-2}\right], D_{\omega}$ crossdiffusion term $\left[\mathrm{Pa} \cdot \mathrm{m}^{-2}\right]$. As our working liquid is water, standard Newton's law was used for modelling its behaviour which allow us to use Womersley number in its standard form:

$$
\alpha=\mathrm{L} \sqrt{\frac{\omega \rho}{\mu}}
$$

where $\alpha$ is the dimensionless Womersley number, $\mathrm{L}[\mathrm{m}]$ is a characteristic length (radius of the tube in our case), $\omega\left[\mathrm{rad} . \mathrm{s}^{-1}\right]$ is an angular frequency, $\rho\left[\mathrm{kg} \cdot \mathrm{m}^{-3}\right]$ is mass density of the working liquid and $\mu$ [Pa.s] is its dynamic viscosity.

\subsection{Setup and Mesh Sensitivity for CFD}

A pure hexahedral mesh of the tube was generated in ANSYS Mesher (ANSYS Inc., USA) with 28 elements with non-uniform size along the radius. Thus the smallest element size of $0.19 \mathrm{~mm}$ was at the wall and the largest size of $0.44 \mathrm{~mm}$ at the central region. Axially, the mesh size was set to $1.8 \mathrm{~mm}$ in the half-length of the tube and gradually refined towards the inlet and outlet, ending here with 0.28 $\mathrm{mm}$. The quarter of the tube was circumferentially divided into 18 elements. The total number of elements was $174 \mathrm{k}$. To check the mesh sensitivity, the mesh was isotropically and uniformly refined to $509 \mathrm{k}$ elements (see Fig. 4). After this mesh refinement, the cross-sectional averaged velocity values in the half-length section of the tube were not influenced (difference between maximum values < $1 \%$ ) and the velocity profiles matched well over the whole period. Although the velocity profiles near the wall were almost identical, a slight delay of BL separation was observed in case of the coarse mesh (0.012 s).

To set the input $\mathrm{BC}$ in the simulation, the velocity profiles were recorded experimentally using 2D PIV and integrated throughout the cross section in each time point $(1000 \mathrm{~Hz})$ to obtain the transient mass flow profile. Because the tube is almost rigid (glass tube), the time shift between the middle and inlet sections of the tube tends to zero. This allows us to use this profile as a mass flow boundary condition at the inlet. At the outlet, the transient pressure profile measured by the lower (output) pressure sensor was used. However, a special care had to be devoted to this pressure profile. The main reason was that although the absolute measured pressure value was relatively large (ca $170 \mathrm{kPa}$ ), the pressure difference (i.e. the pressure loss) between the inlet and outlet of the tube was very small, on the same order as the accuracy of the pressure transducers $(0.35 \%$ of their maximum measurable range of $200 \mathrm{kPa}$, i.e. $700 \mathrm{~Pa}$ ). To eliminate the high frequency noise, a six order Butteworth low pass frequency filter (MATLAB) was applied on the measured profile (Fig. 2A). Time step size was set to $0.004 \mathrm{~s}$, which was confirmed to be sufficient even for the compliant tube (see Chapt. 3.2). To achieve the required convergence criteria for residuals, 20 iterations were used per time step. These criteria were set to $10^{-5}$ for continuity, TKE and specific turbulence dissipation rate $\omega$, and to $10^{-}$ ${ }^{3}$ for velocity components.

\subsection{Setup for FSI Simulations}

In this case, a tube made of silicone (a highly compliant polydimethylsiloxane - PDMS) was measured in the test region and then simulated numerically. Based on three material tests (uniaxial, equbiaxial and pure shear test), performed with our in-house testing machine, the PDMS tube was considered as hyperelastic, isotropic, incompressible and homogeneous. To identify the material model, HYPERFIT software (http://www.hyperfit.wz.cz/) was used and the best fit was achieved for NeoHookean constitutive model with the initial shear modulus of $0.64 \mathrm{MPa}$, which corresponds to Young's modulus of 1.9 MPa.

Viscoelastic properties were neglected because of low $(1 \mathrm{~Hz})$ pumping frequency (Placet and Delobelle 2015). Due to compliance of the PDMS tube, the time shift between the middle and the inlet sections of the tube becomes larger and quite significant. Therefore, the mass flow wave computed from the experimental velocity profiles at the middle of the tube would be highly different from that at the inlet. For this reason, another type of $\mathrm{BCs}$ had to be chosen than that used for the rigid case.

The pressure profiles obtained from the sensors were used as inlet and outlet BCs. The same frequency filter was used for filtering out the high frequency noise as mentioned in chapter 3.1. The time step size was set to $0.004 \mathrm{~s}$ while 8 iterations were used per time step (Fluent) and 5 iterations for coupling the solid and fluid domains. For comparison, the simulation was also performed for the time step of $0.001 \mathrm{~s}$ but the overall differences were small and $\mathrm{CPU}$ time was four times longer. The convergence criteria for residuals were the same as for the rigid case. Dimensions of the tube and range of the velocities are similar for the rigid and compliant cases; hence, the mesh sensitivity study was performed for rigid case only (see chapter 3.1). 


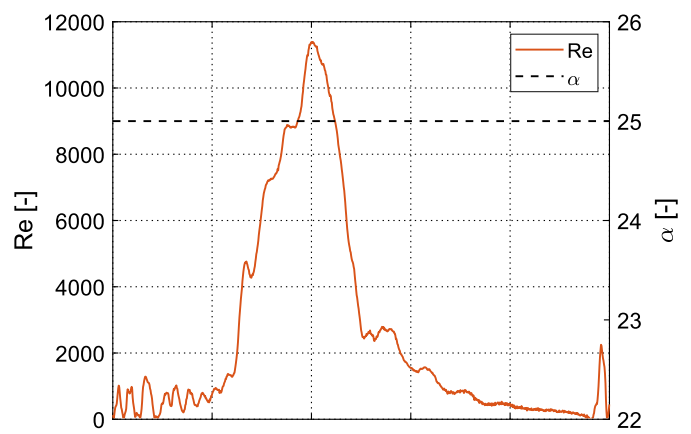

(a)

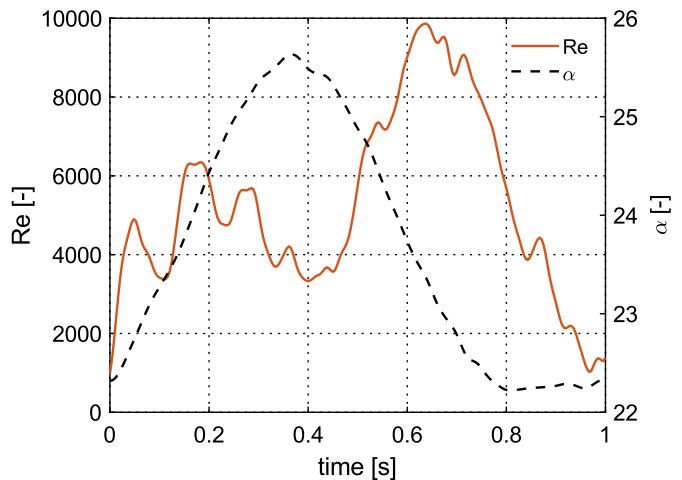

(b)

Fig. 3. Re and $\alpha$ numbers during the period for the rigid (a) and compliant (b) case.

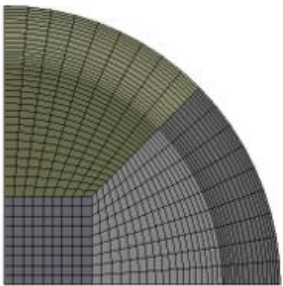

a)
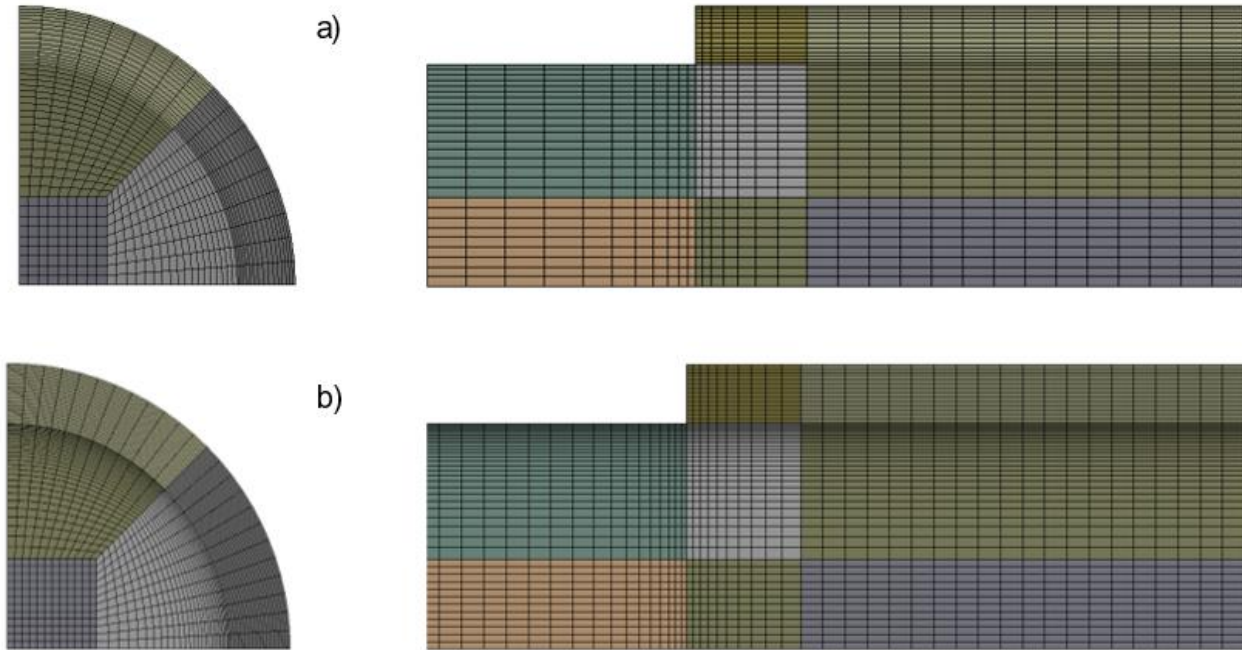

b)

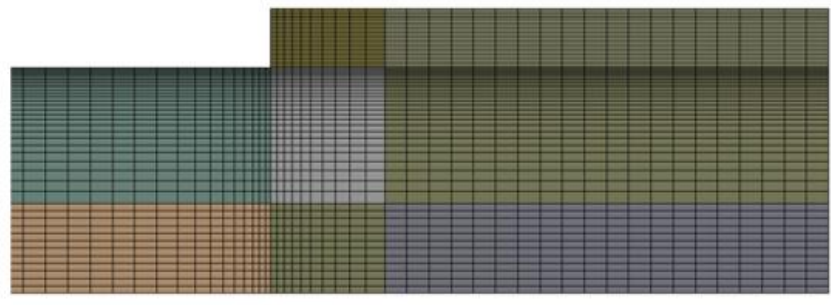

$$
\frac{0}{2,5} \frac{5}{7,5} 10[\mathrm{~mm}]
$$

$\underset{2,5}{0} \frac{5}{7,5} 10[\mathrm{~mm}]$

Fig. 4. (a) Detail of the hexahedral mesh used for computations (174k elements in total); (b) the isotopically refined mesh (509k elements in total).

\section{Results}

To ensure that the size of the element nearest to the wall is sufficient for turbulent model, we checked also the value of $\mathrm{y}^{+}$, which was $<2$ during the whole period for both rigid and compliant cases. For optional low $\mathrm{Re}$ turbulent models like $\mathrm{k}-\omega$ SST/standard it is optimal to have $\mathrm{y}^{+} \sim 1$ (Schultz et al. 2003). To check the impact of this criterion, we refined the mesh up to $509 \mathrm{k}$ elements. With this mesh, the $\mathrm{y}^{+}$values were $<1$ while the results were not significantly changed.

The results were extracted from the $3^{\text {th }}$ and $6^{\text {th }}$ simulated cycle for laminar and turbulent cases respectively, which was sufficient to reach a stabilized cyclic response.

Figure 3 shows development of $\operatorname{Re}$ and $\alpha$ numbers for the rigid and compliant cases. In contrast to the rigid tube with $\alpha \sim 25, \alpha$ is not constant for the compliant tube due to the wall movement, i.e., changes in diameter. Peak values of both $\mathrm{Re}$ and $\alpha$ numbers correspond to the upper part of the physiological range of their values in human healthy ascending aorta (Stein and Sabbah 1976).

\subsection{Rigid tube}

The peak velocities in the middle of the tube are almost identical for data from both simulations and the experiment (Fig. 5A). Also agreement between velocity profiles is very good except for the near wall region (Fig. $6 \mathrm{~A} \div \mathrm{D}$ ). The deviations between turbulent and laminar profiles are negligible which results in almost the same value of WSS except for the late deceleration phase (Fig. $5 \mathrm{~B} ; \mathrm{t} \sim 0.6 \div 0.9$ ). It indicates the flow is laminar during most of the cycle.

By integration of turbulent kinetic energy (ITKE) across the profile of the turbulent model, we can obtain an objective quantification of the amount of velocity fluctuations during the cycle, i. e. of deviations from the laminar flow assumption (Fig. 5A, black dashed curve). Based on the TKE development in time, the period can be divided into four phases. Phase 1 (see Fig. 5A) represents the first half of acceleration in the lower Re region where the 

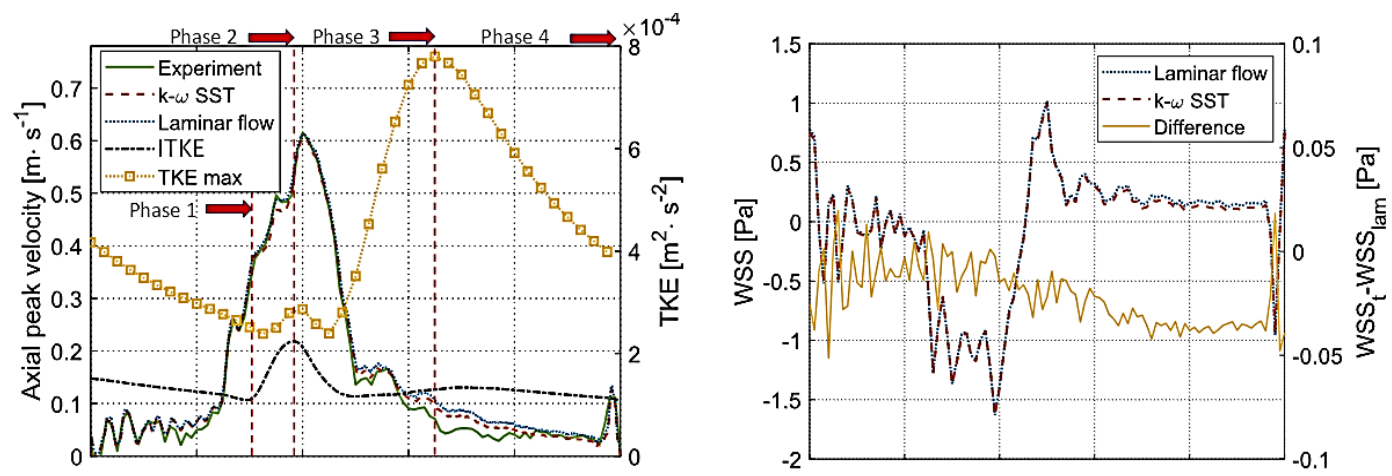

$\times 10^{-4}$
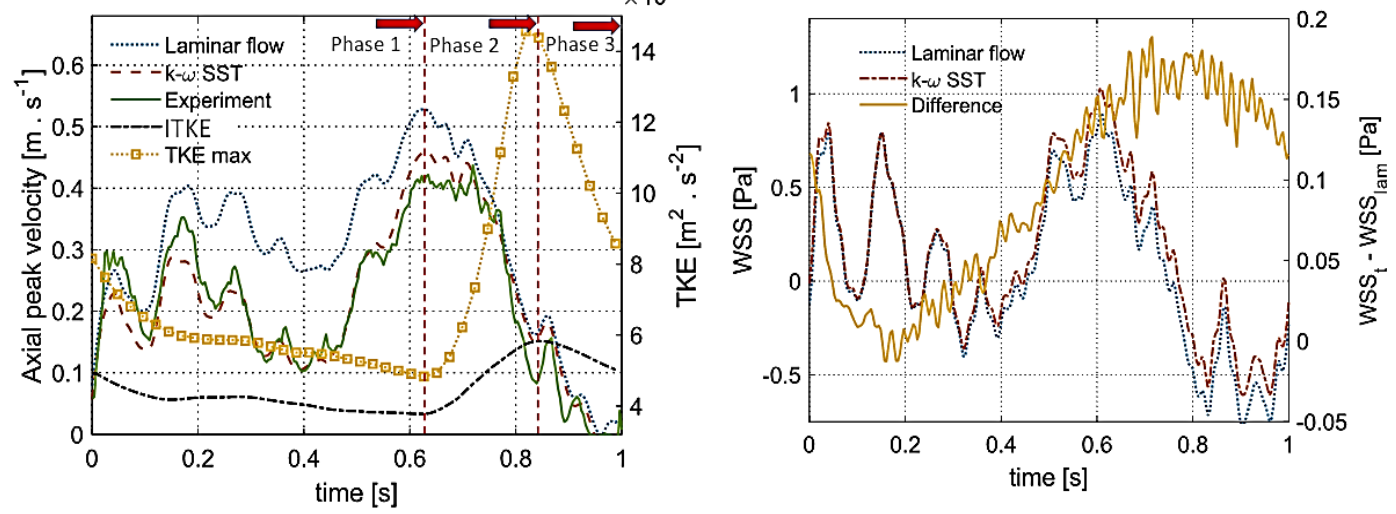

Fig. 5. Time distributions of fluid parameters in the middle of the tube (centre of the cross section at the half-tube length). Left column: axial peak velocity and TKE. Right column: WSS for laminar flow (blue curve), WSS for k- $\omega$ SST turbulent model (orange curve), and the difference between turbulent

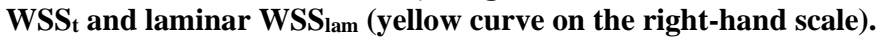

ITKE has a slightly declining trend. Distribution in the lowest point of the ITKE time curve is depicted in Fig. 6A. This phase is in accordance with the stabilization trend of the flow during acceleration. At the beginning of the phase 2, the ITKE starts to increase as a result of two modest slowdowns in velocity during its increase. It is also the only time within the whole period when the TKE spreads significantly to the core (Fig. 6B). During deceleration, the ITKE decreases steeply with velocity. After this rather sudden deceleration (referred to as phase 3, see Fig. 5A), the value of TKE at the flow core tends to zero, but it starts to grow in near wall region (Fig. 6C). This can be explained on the basis of the theory published by (Brindise and Vlachos 2018) that turbulence should most likely occur in the deceleration phase when BL separation occurs. Nevertheless, in this case the deceleration phase is not long enough, thus the turbulence does not develop to the core and higher values of TKE remain only near the wall. In fact, due to the BL separation and the subsequent growth of the back flow, the TKE increases steeply during this phase while the ITKE increase is only very slight (see Fig. 5A; $\mathrm{t} \sim 0,45 \mathrm{~s} \div 0,65 \mathrm{~s}$, the dashed yellow and black curves, respectively). The different slopes of these curves indicate that the TKE increase is only local and occurs in the near-wall region as shown in Fig. 6C and D. In addition, this second peak in the TKE at the near wall region can be caused by the delay between stepped decrease in velocity and progression of turbulence (Brindise and Vlachos
2018). This elevated value of TKE near the wall results in a modest deviation of the turbulent profile from the laminar at this location (see Fig. 6D). Phase 4 starts from $t \sim 0.65 \mathrm{~s}$ when both the ITKE and TKE near the wall decrease again because the velocity tends to zero (see Fig. 5A). As a result, there is not enough energy for development of transitional flow.

\subsection{Compliant tube}

Peak velocities in the middle of the tube from turbulent model simulation are in very good agreement with the experiment during the whole cycle (Fig. 5C). For the laminar simulation, however, a significant offset to higher velocities occurs. We attribute it to its tendency to maintain the parabolic shape (Fig. $7 \mathrm{~A} \div \mathrm{D}$ ). On the other hand, flattening of the turbulent velocity profiles corresponds very well to the experimental PIV data (Fig. $7 \mathrm{~A} \div \mathrm{D}$, orange and green curves, respectively). The difference in WSS (Fig. 5D) tends to zero at the beginning of the period and increases linearly between $\mathrm{t} \sim 0.2 \mathrm{~s}$ and $0.65 \mathrm{~s}$ when it reaches $\sim 0.15 \mathrm{~Pa}$, which corresponds to $\sim 30 \%$ relative deviation. A similar difference lasts until the end of the period while the WSS is changing its orientation; thus the relative deviation is much higher in the time when the WSS is crossing the zero value (at $\mathrm{t} \sim 0.75 \mathrm{~s}$ ). Around this time, the peak values of TKE are located mostly at the wall (Fig. 7C) where their impact on the velocity gradient - and consequently on WSS - is most pronounced. 

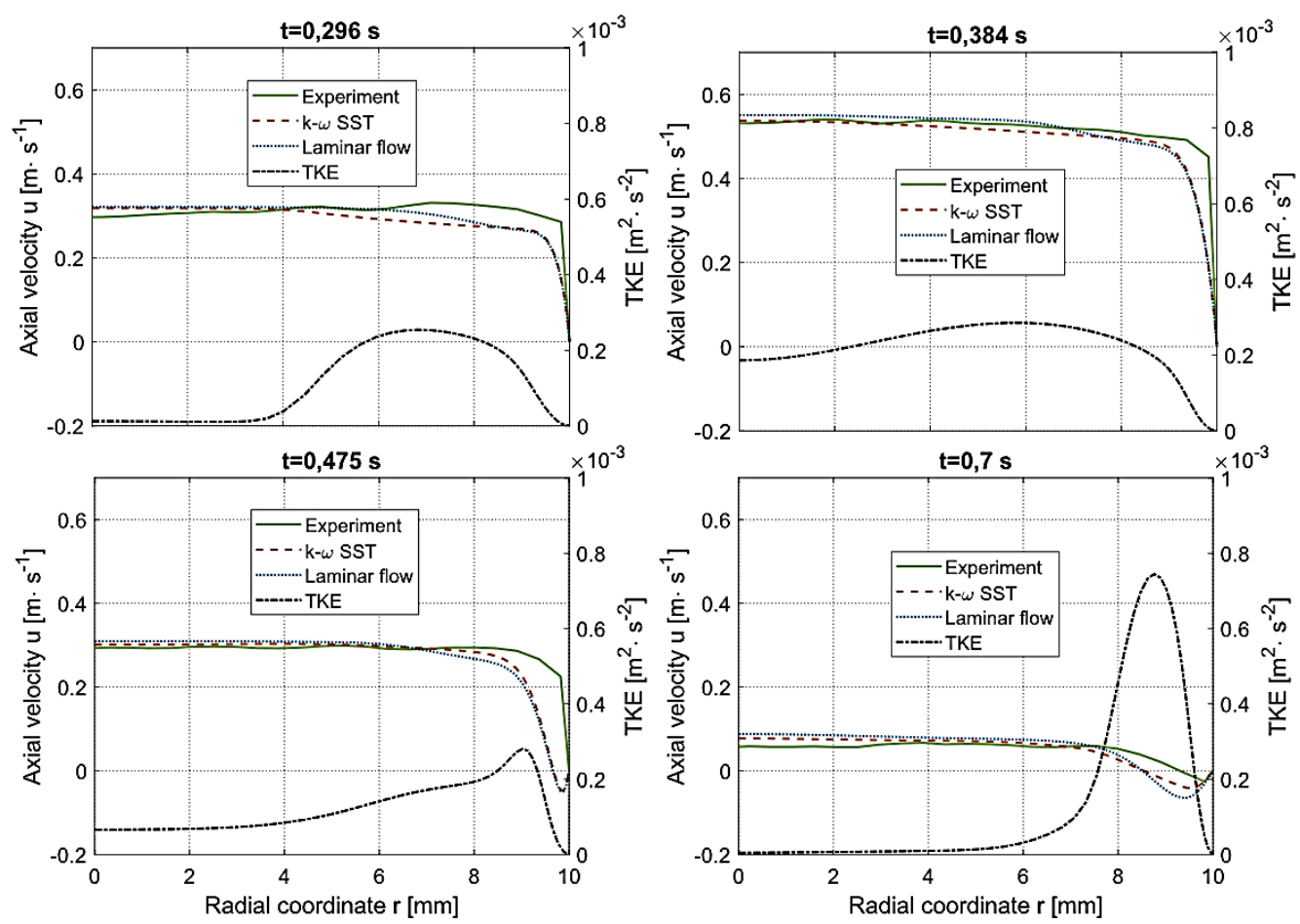

Fig. 6. Velocity profiles compared between experiment, simulation with laminar flow and simulation with low Re turbulent model (here also with TKE) for the rigid tube at the following time points: A) - min ITKE; B) - max ITKE; C) - onset of TKE increase at the wall; D) - max TKE.

The period was divided into parts based on development of the TKE in time, similarly to the rigid tube case. Phase 1 (see Fig. 5C) represents more than the first half of the whole period; the flow oscillates within this phase at higher frequencies and the TKE decreases slowly. It is worth to note that ITKE is more than three times higher than in case of the rigid tube, most probably due to these disturbances (see Fig. 5A and C, black dashed curves). The lowest point of the ITKE curve ( $\mathrm{t} \sim 0.63$ s, see Fig. 5C) ) is depicted across the radius in Fig. 7A. It shows that the TKE spreads already across the whole radius, which results in a high deviation between the turbulent and laminar velocity profiles. The flow stabilizing process induced by the acceleration is probably stronger than in the rigid case and remains unaffected by the modest slowdown in velocity (Fig. $5 \mathrm{C}, \mathrm{t} \sim 0.5 \div 0.55 \mathrm{~s}$ ). During the phase 2, ITKE increases by more than $50 \%$ of its minimum value. Similarly, the maximal TKE value across the radius increases even more steeply (almost three times - Fig. 5C). Regarding to the radial position, the TKE starts to increase in the near wall region (Fig. 7B) and continues to increase here up to its maximum (Fig. 7C). The ITKE peak is slightly delayed after the peak TKE value across the radius (see Fig. 5C). Discrepancies between the turbulent and laminar velocity profiles at this peak (Fig. 7C) are significant, especially in the region near the wall. Phase 3 starts as soon as the TKE values (both the ITKE and the maximum TKE across the radius) begin to decrease. Although the velocity decreases sharply and tends to zero, the ITKE decreases only slightly. This is an opposite trend as for the rigid case, where ITKE decreased with velocity to the pre-pulse values. This difference should be attributed to duration of the deceleration phase, which is longer for the compliant tube and thus provides time enough for the transition to turbulence, i.e. spreading of the TKE from the near wall region to the flow core (Fig. 7C and D). The most prominent difference between the turbulent and laminar velocity profiles near the end of the period represents a consequence of this effect (Fig. 7D).

\section{DISCUSSION}

The overall agreement of the velocity profiles between experiment and simulation with the turbulent model is very good for both cases. Discrepancies in the near wall region are mainly due to the relatively coarse radial resolution of the camera. Since the dynamic range and spatial resolution of this method is limited by the size of the camera's chip, higher small-scale resolution is always accompanied by losing large-scale picture. Takeuchi et al. (2005) overcame this issue by dividing the measurement region into two sections. Such configuration allows one to perform for a steady flow two separate measurements: One of them with a higher resolution to capture small-scale structures near the wall and another one with a lower resolution to cover all the velocity profile. However, the experiment performed here described the dynamic of unsteady flow which was recorded continuously. Therefore it could not be performed as two separate measurements (with higher and lower resolution) with their average flow fields put together. Since our study aims at comparison of two modelling techniques, the large-scale picture appears sufficient; it shows the flattening of the velocity 

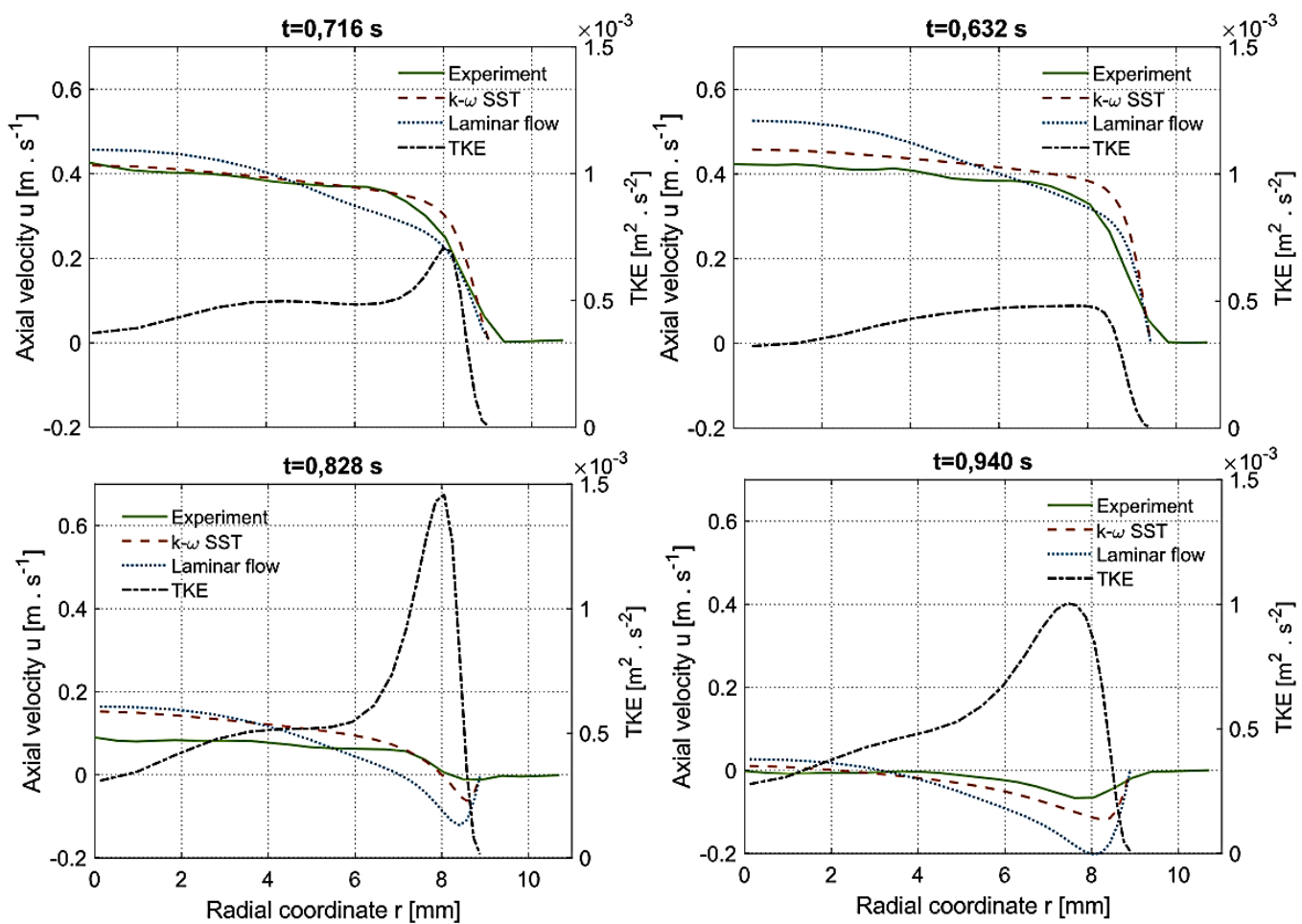

Fig. 7. Velocity profiles compared between experiment, simulation with laminar flow and simulation with low Re turbulent model (here also with TKE) for the compliant tube at the following time points: (A) min ITKE (i.e. maximal velocity); (B) onset of TKE increase at the wall; (C) max TKE; (D) end phase of the period.

profiles, as well as a secondary gradient near the wall. A sufficient quality of the image near the wall was reached due to the application of fluorescent particles; in this way, the laser light scatter induced by the wall is reduced by the lens band-pass filter (580nm wavelength) and the measurements close to the wall are more precise.

Comparison of Fig. 5A and $\mathrm{C}$ shows that the velocity waveforms in the middle of the tube differ significantly. As the experimental setup and dimensions are almost the same for both, this difference could be mistakenly attributed to the wall movement of the PDMS tube. However, the interpretation cannot be so straightforward. The main reason for such a great discrepancy is the nonnegligible time shift between the pressure waves at the inlet and the outlet, resulting in a totally different pressure gradients along the tube. In addition, at least two locations exist in case of the compliant tube where an almost stepwise change in the impedance occurs. They are located at the connections between the tested tube and the hydraulic circuit where the pressure sensors are embedded in metal (i.e. nearly rigid) casings (see Fig. 1C) and are responsible for a high portion of the reflected waves. This effect could be diminished by using materials with similar elastic modules for the whole circuit. Another source of the wave reflections and consequent oscillations, valves of the membrane pump, could be eliminated by using a peristaltic pump instead.

The closed experimental circuit with its stepwise changes of stiffness is inconsistent with the arterial tree, which is characterized by a continuous distribution of parameters and terminated with a constant pressure in the capillary bed; thus it does not induce as many wave reflections as it was in the experimental circuit with the stepwise changes of stiffness. However, from the validation point of view, our conditions were harder (high frequency oscillations) than may occur in FSI simulations of blood flow. Therefore, the conclusions drawn on the basis of our simulations should be valid also for simulations of arterial flow, which can be, in addition, influenced also by non-Newtonian blood properties and strain stiffening of the arterial wall.

In our study we worked with a relatively well described tube (homogeneous, isotropic and hyperelastic) and also the boundary conditions were recorded. However, for many computational studies which consider compliance of blood vessels (FSI problems), such comprehensive inputs are unknown. The number of required inputs can be reduced by lumped parameter model (Westerhof et al. 2009) or structured tree model (Olufsen et al. 2000), usually used instead of the measured output pressure. For instance, in case of the lumped three-parameter Windkessel model used instead of the recorded transient pressure waveform for the outlet, only three unknown constants have to be determined, i.e., peripheral compliance, resistance and inertance. Another easy option is to adopt representative BCs from the available literature. In this case, however, a synchronization between the input and outputs has to be done for correction of the time shift in the computational model. If not done, the pressure gradients along the domain can be highly different 
from their physiological values. It may result in a non-physiological flow in the simulations and, consequently, in incorrect conclusions. We referred here some of them (Xenos et al. 2010; Gao et al. 2013; Drewe et al. 2017) where no correction was done and the results should be regarded very carefully and more critically for that reason.

Validation of our simulations was performed via experimental data from 2D PIV (velocity profiles); the agreement was excellent for the rigid tube and very good for the compliant case simulated using the turbulent model. In both cases a significant stabilizing effect on the flow was observed during the acceleration phase. For the rigid case, the laminar flow model appears sufficient due to the lack of the TKE, which would be needed for spreading the turbulence. The main reason consists in the very short, almost stepped deceleration phase, which cannot provide time enough for development of turbulent structures. On the other hand, the deceleration phase lasts longer in the compliant tube and also the pre-pulse level of the TKE is higher. Under these circumstances the TKE spreads from the near wall region to the core flow. As a result, the differences between laminar and turbulent velocity profiles become significant, especially during the deceleration phase. The same trend can be regarded for WSS. Turbulent profiles correspond quantitatively and qualitatively very well to the PIV data. As this is not true for laminar flow model, we conclude that it is not suitable here.

Although both simulations and experiments were performed with water, the dimensionless numbers (peak $\operatorname{Re}$ and $\alpha$ ) were chosen in upper part of the physiological range measured in vivo in healthy human ascending aorta. In contrast to water, human blood behaves as a non-Newtonian liquid. Red blood cells, as the main source of non-Newtonian behaviour, may aggregate into groups (rouleaux). In an equilibrium state or at sufficiently small shear rates, blood behaves like a solid until a threshold in shear stress (i.e. yield stress) is reached. The threshold lies between 0.0015 and $0.005 \mathrm{~Pa}$ for healthy human blood (Fedosov et al. 2011). On the other hand, for large shear rates the rouleaux are dispersed almost completely into individual red blood cells and the blood behaves like a Newtonian liquid. This is the case of larger arteries where this simplification is broadly applied (Miyazaki et al. 2017; Drewe et al. 2017; Gao et al. 2013; Xenos et al. 2010) and should not induce significant errors.

\section{Conclusion}

Simulations of the pulsatile flow in rigid and compliant tubes were performed and validated with experimental data from 2D PIV. For the rigid case, the laminar flow model appears sufficient while for the compliant tube the laminar velocity profiles differ significantly from the turbulent and experimental profiles, especially during the deceleration phase. Therefore, the turbulent k- $\omega$ SST model should be preferred for compliant tubes. However, the differences should not be attributed only to the direct effect of the wall movement but also to its influence through secondary factors, e.g. a longer deceleration phase, a totally different distribution of pressure gradients along the tube and the stepwise changes in impedance between the compliant tube and the casings of pressure sensors. The length of the deceleration phase within the velocity profile measured in human vessels should be considered as an important factor regarding to the character of flow and other relevant outputs such as WSS and other hemodynamic parameters. Nevertheless, determination of a critical length of the deceleration phase will be subject of further research. Also the impact of strain stiffening typical for arterial tissues and of non-Newtonian behaviour of blood remains out of scope of this study and should be addressed in future.

\section{ACKNOWLEDGEMENTS}

This work was supported by Czech Science Foundation, projects No. 17-19444S and No. 1813663S.

\section{REFERENCES}

Brindise, M. C. and P. P. Vlachos (2018). Pulsatile Pipe Flow Transition: Flow Waveform Effects. Physics of Fluids 30(1).

DiCarlo, A. L., D. W. Holdsworth and T. L. Poepping (2019). Study of the Effect of Stenosis Severity and Non-Newtonian Viscosity on Multidirectional Wall Shear Stress and Flow Disturbances in the Carotid Artery Using Particle Image Velocimetry. Medical Engineering and Physics 65, 8-23.

Drewe, C. J., L. P.Parker, L. J. Kelsey, P. E. Norman, J. T. Powell and B. J. Doyle (2017). Haemodynamics and Stresses in Abdominal Aortic Aneurysms: A Fluid-Structure Interaction Study into the Effect of Proximal Neck and Iliac Bifurcation Angle. Journal of Biomechanics 60, 150-56.

Fedosov, D. A., W. Pan, B. Caswell, G. Gompper and G. E. Karniadakis (2011). Predicting Human Blood Viscosity in Silico. Proceedings of the National Academy of Sciences 108(29), 11772-77.

Gao, F., H. Ueda, L. Gang and H. Okada (2013). Fluid Structure Interaction Simulation in Three-Layered Aortic Aneurysm Model under Pulsatile Flow: Comparison of Wrapping and Stenting. Journal of Biomechanics 46(7), 1335-42.

Mcdonald, D. A. and M. College (1954). The Relation of Pulsatile Pressure to Flow in Arteries. Journal of Physiology 127, 533-52.

Miyazaki, S. K. Itatani, T. Furusawa, T. Nishino, M. Sugiyama, Y. Takehara and S. Yasukochi (2017). Validation of Numerical Simulation Methods in Aortic Arch Using 4D Flow MRI. Heart and Vessels 32(8), 1032-44. 
Jagos et al. / JAFM, Vol. 14, No. 5, pp. 1411-1420, 2021.

Morris, P. D., D. A. Silva Soto, J. F. A. Feher, D. Rafiroiu, A. Lungu, S. Varma, P. Lawford, V., D. R. Hose and J. P. Gunn (2017). Fast Virtual Fractional Flow Reserve Based Upon SteadyState Computational Fluid Dynamics Analysis: Results From the VIRTU-Fast Study. JACC: Basic to Translational Science 2(4), 434-46.

Olufsen, M. S. C. S. Peskin, W. Y. Kim, E. M. Pedersen, A. Nadim and J. Larsen (2000). Numerical Simulation and Experimental Validation of Blood Flow in Arteries with Structured-Tree Outflow Conditions. Annals of Biomedical Engineering 28(11), 1281-99.

Özahi, E. and M. Ö. Çarpınlığlu (2017). A NonDimensional Oscillation Parameter Describing Interactive Influence of Oscillation Frequency and Velocity Amplitude Ratio for Use in Pulsatile Flows. Measurement: Journal of the International Measurement Confederation 99, 36-43.

Placet, V. and P. Delobelle (2015). Mechanical Properties of Bulk Polydimethylsiloxane for Microfluidics over a Large Range of Frequencies and Aging Times. Journal of Micromechanics and Microengineering 25(3).

Schultz, M. P., J. A. Finlay, M. E. Callow and J. A. Callow (2003). Three Models to Relate Detachment of Low Form Fouling at Laboratory and Ship Scale. Biofouling 19(SUPPL.) 17-26.
Stein, P. D. and H. N. Sabbah (1976). Turbulent Blood Flow in the Ascending Aorta of Humans with Normal and Diseased Aortic Valves. Circulation Research 39(1), 58-65.

Takeuchi, J., S. Satake, N. B. Morley, T. Yokomine, T. Kunugi and M. A. Abdou (2005). PIV Measurements of Turbulence Statistics and Near-Wall Structure of Fully Developed Pipe Flow at High Reynolds Number. 6th International Symposium on Particle Image Velocimetry Pasadena, California, USA, September 21-23: 1-9.

Westerhof, N., J. W. Lankhaar and B. E. Westerhof. (2009). The Arterial Windkessel. Medical and Biological Engineering and Computing 47(2), 131-41.

Xenos, M., Y. Alemu, D. Zamfir, S. Einav, J. J. Ricotta, N. Labropoulos, A. Tassiopoulos and D. Bluestein (2010). The Effect of Angulation in Abdominal Aortic Aneurysms: FluidStructure Interaction Simulations of Idealized Geometries. Medical and Biological Engineering and Computing 48(12), 1175-90.

Xu, D. et al. (2017). Transition to Turbulence in Pulsating Pipe Flow. Journal of Fluid Mechanics 831, 418-32.

$\mathrm{Xu}, \mathrm{D}$. and M Avila (2018). The Effect of Pulsation Frequency on Transition in Pulsatile Pipe Flow. Journal of Fluid Mechanics 857, 93751. 